\title{
Orientation marché et innovation collaborative par les interactions université-industrie
}

\author{
Market orientation and collaborative innovation through university-
} industry interactions

\author{
Cheikh Abdou Lahad Thiaw ${ }^{1}$ \\ ${ }^{1}$ École Supérieure Polytechnique (ESP)/Université Cheikh Anta Diop de Dakar | GREDEG CNRS/Université Côte d'Azur \\ cheikh.thiaw@gmail.com | cheikh.thiaw@esp.sn | cheikh.thiaw@univ-cotedazur.fr | cheikh.thiaw@gredeg.cnrs.fr
}

RÉSUMÉ. Le développement incessant des réseaux d'innovation (clusters, pôles de compétitivité) a forcé des évolutions majeures dans les procédés de collaboration entre acteurs économiques et ceux de la recherche académique. Par la mise sur l'innovation, ces dispositifs hétéroclites sont les outils de propulsion du succès des économies de demain et suscitent un grand intérêt auprès des chercheurs et praticiens, mais aussi auprès des États. Toutefois si leur propicité aux innovations n'est plus à démontrer, l'absence d'une approche marketing et orientation marché des projets collaboratifs limite leur portée (diffusion et adoption par le marché). Ces difficultés sont notamment liées aux intentions stratégiques différenciées des acteurs et leur défaut de visions partagées autour des aspects "marché ». Cet article examine les apports d'un marketing de l'innovation appliqué dans un cadre d'interaction collaborative entre entreprises et universités et questionne la problématique du dilemme de l'innovation "Market-pull» "Techno-push». II préconise d'octroyer un rôle central au marketing en tant que fonction transversale des projets d'innovation collaboratifs, avec le marché comme fil conducteur de ses actions et vecteur d'intégration de l'innovation. Nous considérons que l'innovation collaborative performante est basée sur l'adoption d'une approche hybride privilégiant la dimension technologique tout en intégrant les aspects « marché ».

ABSTRACT. The constant development of innovation networks (clusters and other related concepts) has forced major changes in the collaboration practices between firms and academics. By promoting innovation, these heterogeneous systems are considered to be the necessary tools for propelling the success and competitiveness of the economies of tomorrow. They are attracting growing interest from researchers and practitioners, but they also generate interest from governments. However, while they are very conducive to generating innovations, the lack of a marketing and marketoriented approach of collaborative projects within them limits their scope (dissemination and market adoption). These difficulties are notably linked to the consequences of the diversity of stakeholders' strategic intentions and their lack of shared visions about the "market" aspects. This article examines the contributions of innovation marketing, applied to a collaborative context with interactions between companies and academics. It investigates the "market-pull" - "technopush" innovation dilemma. It advocates giving marketing a key role as a transversal function within collaborative innovation projects, with the market as the common thread of its actions and a vector for the integration of innovations. We consider that successful collaborative innovation is based on a hybrid approach, focusing on the technological dimension while integrating market aspects.

MOTS-CLES. Marketing de l'innovation, Innovation collaborative, Clusters, Orientation client, Marché, Technologie. KEYWORDS. Innovation marketing, Collaborative innovation, Clusters, Customer orientation, Market, Technology.

\section{Introduction}

L'économie mondiale déstructurée a forcé l'élaboration de nouvelles politiques d'appui au développement basées notamment sur la promotion de l'innovation dans des réseaux prenant la forme de clusters [POR 90, POR 98, POR 00, POR 04], de parcs technologiques, de pôles de compétitivité [CHAB 09, CHA 10] et d'autres types d'écosystèmes et de configurations connexes [THIa 17]. Ces dispositifs se caractérisent par la congrégation d'acteurs d'horizons différents, l'impulsion de mécanismes de travail collaboratifs et la mise sur l'innovation pour renforcer les performances industrielles et stimuler la compétitivité des pays. Grâce aux perspectives d'innovation qu'ils permettent, ces concepts représentent les outils de propulsion du succès des économies de demain et ont suscité un grand intérêt auprès des chercheurs, des praticiens et des pouvoirs publics. C'est dans ce contexte que les États promeuvent de plus en plus les relations de collaboration entre les acteurs (C) 2021 ISTE OpenScience - Published by ISTE Ltd. London, UK - openscience.fr 
académiques (universités) et le milieu industriel (entreprises) pour promouvoir la génération d'innovations commercialisables et susceptibles d'une création de valeurs économiques. Et c'est de là qu'a émergé le concept d'innovation collaborative en tant que résultante de l'évolution forcée des procédés de collaboration entre acteurs économiques et ceux de la recherche académique [THI 13, THI 16, THI 18].

Toutefois, la littérature a montré que ces dispositifs hétéroclites renferment certes les facteurs de propulsion de l'innovation, mais l'absence d'une approche marketing et orientation marché des projets collaboratifs limiterait la portée et l'effectivité de leurs résultats (diffusion et adoption des innovations). En effet, dans pareils milieux à la fois hétérogènes et interorganisationnels, et requérant une collaboration entre acteurs de natures et d'objectifs différents, voir même antinomiques à bien des égards (entreprises et universités), l'objectif d'inonder le marché d'innovations a parfois paru utopique. Une trop forte focalisation sur les technologies occasionne un «déficit de réalisations par rapport aux attentes pour l'accès facilité à de nouveaux marchés/clients potentiels » [BEA 12].

Ce constat nous autorise à poser la problématique de l'orientation marché au sein de ces outils d'interaction entre acteurs académiques et entrepreneuriaux (université-industrie). Les relations portant souvent sur le processus d'innovation et les activités de R\&D collaboratives, elles concèdent aussi de se questionner sur les dilemmes soulevés par les praticiens à propos de la complexité de l'innovation : doit-on privilégier les opportunités technologiques ou viser prioritairement la réponse aux attentes du marché ? Faut-il pousser l'innovation technologique plutôt que préférer l'élaboration de nouveaux modèles économiques? Le cadre théorique concerné renvoie à la question du dilemme de l'innovation market-pull, techno-push.

Ainsi, ce texte explore les perspectives du marketing de l'innovation à l'échelle interorganisationnelle, et il questionne la problématique du dilemme « Market-pull » «Techno-push » dans un contexte d'innovation collaborative. La question de l'innovation dans les entreprises a été largement évoquée (production, processus, $R \& D$ ), mais celle d'un marketing de l'innovation mettant l'accent sur l'adoption et la commercialisation des innovations issues de la collaboration universitésindustries n'a pas encore reçu un intérêt prononcé de la part des chercheurs. En effet, la plupart des recherches et les définitions amorcées sont confinées dans des thématiques traitant uniquement de sujets liés aux produits [OEC 05].

Les difficultés de diffusion et d'adoption des innovations générées dans les réseaux hétérogènes sont liées aux intentions stratégiques diversifiées des acteurs et le défaut de visions partagées autour des aspects «marché » des projets. Dans ce cadre et afin de parer à ces écueils, être efficace et se constituer en un générateur d'innovations qui soient prêtes à l'accès aux marchés, le processus d'innovation collaborative a besoin du marketing comme une fonction de centralisation de ses interactions, à travers l'orientation marché. Nous considérons en effet que le marketing doit jouer un rôle de chef d'orchestre afin de permettre aux possibilités permises par la technologie de matcher avec les réalités du marché, en adoptant une approche hybride située entre les stratégies d'innovation market-pull et techno-push. C'est de cette manière que l'innovation collaborative pourrait se constituer en source de performance pour les entreprises et fondements de compétitivité pour les économies.

Outre la question de l'adoption des innovations issues de milieux hétérogènes, cette recherche permet aussi d'ouvrir d'autres grilles d'analyse portant sur des business modèles d'innovation spécifiques aux réseaux et sur les mécanismes d'introduction d'une vision marché et entreprise dans les universités.

Notre texte porte sur le marketing de l'innovation en plaçant le curseur sur le concept d'orientation marché [DAY 91, DAY 94 ; NAV 90 ; KOH 93, KOH 90 ; RUE 92 ; DES 93], mais analysé dans un contexte de collaboration interorganisationnelle [THI 13]. Enfin, il procure un regard contemporain sur l'innovation selon un point de vue marketing. 


\section{Marketing de l'innovation et collaboration interorganisationnelle}

\subsection{De l'innovation collaborative imposée...}

Dans une économie moderne marquée par l'apparition constante de produits et de technologies novateurs ainsi qu'une rapide compression des cycles de vie des technologies, l'innovation apparaît comme la solution idéale, la source de croissance pertinente que les organismes et les États se doivent de privilégier. En effet, miser sur l'innovation procure à l'entreprise des ouvertures de marché, des avantages concurrentiels et des potentialités de développement incommensurables. De même, cela permet aux États de propulser leur développement et d'impulser une croissance économique pérenne et inclusive. Pour en tirer pleinement profit et faire face aux aléas néfastes de la mondialisation, les pouvoirs publics ont déployé de nouvelles formes de politiques d'appui au développement basées sur la promotion de l'innovation collaborative par les interactions entre industriels (grandes entreprises, PME et start-up) et universitaires (universités, organismes de formation privés, laboratoires de recherche et centres de recherche privés) autour d'activités de R\&D [POR 90, POR 98, POR 00, POR 04 ; BEL 05 ; RET 09 ; CHAB 09, CHA 10 ; GOS 10 ; THI 13, THI 18 ; CRE 15 ; TOR 15, VIC 16, VON 16].

Pour concrétiser ces différentes politiques, les orientations stratégiques adoptées et les supports d'opérationnalisation conçus prennent des configurations et des dénominations disparates. L'étude de la littérature en la matière fait ressortir plusieurs classifications. À titre d'illustration, nous distinguons une typologie des réseaux basée soit sur une orientation production soit une orientation innovation (systèmes territoriaux de production et systèmes territoriaux d'innovation [CHAB 09; MAI 03]), ou encore d'autres modèles de territoires d'innovation comme les Systèmes de Production Locaux [AYD 86], les «Broader Systems of Innovation» [EDQ 97], les «Regional Learning Economy» [COO 98] et les «nouveaux espaces industriels » [STO 88 ; SAX 94]. Dans le cadre de cet article, nous nous limitons à une distinction plus récente évoquant des réseaux de profil «top-down » et d'autres de profil «bottom-up» [THI 13]. Les premiers, desquels fait partie le modèle des pôles de compétitivité français créé par des initiatives politiques portées par le gouvernement français [THI 13 ; CHA 13] sont caractérisés par l'existence d'un rôle important des autorités publiques aussi bien dans leur mise en place que dans leur fonctionnement. Les secondes, parmi lesquels nous citons les districts industriels [MAR 90], les clusters [POR 90 ; BEL 05] ou encore les écosystèmes d'affaires [RET 09], se particularisent à travers un rôle davantage prégnant des acteurs industriels, mais aussi des scientifiques (universités, laboratoires de recherche), aussi bien dans leur émergence que dans leur mise en œuvre [THI 13, THI 18]. Le cluster de la Silicon Valley (USA) est l'un des exemples les plus représentatifs de ce deuxième groupe, car né de l'action conjointe de l'Université de Stanford en Californie (celle de son Vice-Président, Fred Terman) et des entrepreneurs William Hewlett et David Packard (fondateur de la célèbre marque informatique HP - Hewlett Packard).

De manière similaire, les deux modèles de configuration de réseaux octroient une place de choix à l'innovation, et imposent en quelque sorte une approche d'innovation collaborative basée sur des interactions universités-industries. Dans le champ des travaux qui traitent de leurs performances, plusieurs indicateurs sont mis en évidence. Ils portent notamment sur leurs mécanismes de gouvernance [BOC 06, BOC 09 ; LOU 08, LOU 11], leur composition en termes de profits d'acteurs [CHA 09], l'implication des parties prenantes à leur sein [THI 13] ou encore la nature des projets d'innovation qui y sont conduits [VER 08].

Toutefois, l'innovation collaborative et sa réussite ne se décrètent pas, ni par des initiatives politiques ni par une simple volonté de partenaires décidés à s'unir pour innover conjointement. Elle est effectivement confrontée aux réalités du terrain et à l'effectivité des profils et des pratiques différentiés des acteurs impliqués, et qui représentent la cause de multiples cas d'échecs de processus d'innovation collaborative, notamment dans les phases finales de diffusion et d'adoption par les marchés [THI 13]. Ces difficultés de diffusion et d'adoption sont surtout liées aux conséquences 
induites par les différences d'intentions stratégiques des acteurs diversifiés, de même que l'absence de visions partagées sur les problématiques liées aux attentes du «marché » des projets collaboratifs.

De ce fait, si ces réseaux collaboratifs présentent certes des prédispositions propices à l'innovation, l'absence d'une approche marketing à travers une orientation marché des projets collaboratifs limite la portée de leurs résultats. C'est la nature antinomique des parties prenantes (entreprises et universités) et leurs disparités mentionnées ci-avant qui empêchent très souvent les innovations générées d'avoir un accès aux marchés et aux usagers susceptibles d'être intéressés [BEA 12; THI 13].

D'où, la pertinence de l'adoption d'une approche tenant compte des réalités de ces milieux, caractérisés également, à la fois par des mécanismes de «socialisation» (au sens de Granovetter [GRA 85]) résultant des interactions des acteurs, et d'un ensemble de composants relatifs à des normes, valeurs, cultures et d'institutionnalisations sociales. Dans ce cadre, il convient de noter que cette forme d'encastrement au sein des réseaux est aussi leur force première, car ils sont avant tout conçus pour valoriser la complémentarité de compétences et de savoir-faire d'acteurs aux natures différentes, en stimulant des mécanismes de génération d'externalités positives et permettre de bénéficier d'économies d'échelles [DEF 10]. Elle est même considérée comme favorable à la logique exploratoire et à la « sérendipité » (le rôle du hasard dans la découverte et les processus d'innovation) en particulier dans l'innovation ouverte [CHE 03]. Dès lors, ce sont moins les visions différenciées qui constituent les causes d'échec de l'innovation collaborative, mais au contraire, ce sont plus les comportements antinomiques des acteurs et les actions divergentes qui interpellent quant au communautarisme épistémique nécessaire à la performance de l'innovation collaborative, par exemple, la trop forte focalisation de certains acteurs sur les technologies au détriment des aspects marché [BEA 12]. C'est ce qui explique la nécessité de création de liants entre marketing de l'innovation et collaboration interorganisationnelle.

\section{2. ...au besoin d'une approche marketing spécifique}

Le marketing de l'innovation fait référence aux techniques marketing spécifiquement utilisées pour s'adapter à cet environnement moderne, complexe et fortement marqué par la prédominance de l'innovation. Il permet aux entreprises d'avoir une maîtrise plus aisée des actions de diffusion des innovations (nouveaux produits, services) grâce à une compréhension plus fine des attentes du marché et une meilleure facilitation de l'adoption des innovations par les clients finaux (consommateurs et utilisateurs).

Dans cette ère de l'économie marquée par la promotion de l'innovation dans des réseaux d'interactions entre industries et universités, le marketing de l'innovation s'invite au débat littéraire pour investiguer la question et tenter de trouver des solutions efficaces aux problèmes de diffusion et d'accès aux marchés des innovations, en quelque sorte de leur commercialisation [BRE 15].

De manière générale, les innovations de produits et de services novateurs, constamment introduites sur les marchés, font l'objet d'une incertitude prononcée et d'un risque de non-adoption, voire de rejet, pour certaines d'entre elles, de la part des consommateurs. Par conséquent, il est important pour les entreprises de porter une attention particulière aux étapes du processus des innovations relatives à leur diffusion, afin d'assurer une commercialisation correcte, le gain d'un succès et la création de valeur pour l'organisme innovateur. Dans le cas de l'innovation collaborative agrémentée d'activités de R\&D, ces risques et incertitudes sont encore plus présents, car la nature hétérogène des acteurs qui interviennent durant le processus complexifie la portée de l'applicabilité des innovations et leur adoption par le marché. Au-delà du concept d'innovation collaborative, différents travaux de recherche actuels portant sur l'innovation dans ce domaine permettent de faire émerger d'autres appellations précurseurs qui interpellent désormais la littérature en marketing : innovation en réseau, innovation ouverte ou fermée, innovation interne ou externe... 
De manière globale, l'innovation par la R\&D implique le choix entre deux options tactiques: prioriser le marché (market-pull ou market-driven) ou privilégier la technologie (technology-push ou techno-push). Pour le cas des réseaux dans lesquels l'innovation est le fruit d'interactions universitésentreprises, ce dilemme d'orientation marché et/ou de focalisation sur la technologie est encore plus pesant. Dans ces cas de figure en effet, l'innovation est générée par des activités dont les périmètres d'intervention sont occupés par des acteurs de natures et d'intentions stratégiques différentes. Ces derniers évoluent dans des milieux aux valeurs culturelles et aux critères d'évaluation de performances différents, voire antinomiques.

Dans les laboratoires de recherche universitaire, les chercheurs mettent au point des innovations par la conversion de leurs connaissances scientifiques (invention) sans se soucier, au préalable, des attentes du marché. En effet, leur objectif premier est de produire de nouvelles connaissances scientifiques indépendamment d'une recherche de perspectives d'application. L'évaluation de leurs performances s'intéresse prioritairement à la portée de leurs résultats en matière de publications scientifiques et de reconnaissance (attribution de prix, par exemple). Pour cela, elle s'appuie sur des indicateurs bibliométriques pour évaluer la production scientifique en ayant recours à des critères d'impacts des revues.

Alors qu'au sein des entreprises, ce sont plus les utilisateurs - et non les producteurs — qui sont à l'origine des innovations [THI $11 ; \mathrm{JOH} 11]$. C'est-à-dire que pour les industriels, l'innovation procède d'abord de la satisfaction des besoins des utilisateurs finaux, c'est-à-dire le marché. Leur performance dépend de la commercialisation et des volumes de ventes réalisées.

Dès lors, les objectifs d'orientation et de performance des entreprises peuvent être distantes des intérêts scientifiques alors que les chercheurs universitaires peuvent aussi être éloignés des notions d'orientations marché. Ainsi, les intentions stratégiques différenciées des acteurs de l'industrie et de l'université impliquent des comportements disparates qui influent sur les choix d'orientations stratégiques des projets d'innovation collaboratifs. Du coup, en fonction de leur importance dans les projets et la considération du poids de leurs représentants respectifs, la génération d'innovations propices à l'accès aux marchés peut être impactée.

Ces dernières années, de nombreux travaux ont été dédiés au travail en connexion. Cependant, les thématiques abordées sont uniquement orientées sur les interactions des acteurs et la performance des réseaux d'innovation, donc sur des mécanismes de fluidification et d'amélioration du travail des acteurs de l'innovation collaborative. Elles ont plus investigué les phases de production conjointe des connaissances d'innovation, et moins analysé les manœuvres postérieures et les pratiques postinnovations et relatifs à la diffusion sur les marchés et la commercialisation des innovations générées [THI 13]. C'est ce qui explique l'échec de la première phase des pôles de compétitivité français (20052008), car l'encastrement de savoirs et de savoir-faire ne suffit pas pour permettre aux relations industries-entreprises d'être efficientes en matière de potentiel d'innovation [THI 09]. Il faut en parallèle favoriser l'émergence de compétences de «savoir vendre» indispensables au succès du processus d'innovation et l'adoption des innovations par le marché. L'innovation est certes très importante et reste indispensable pour la pérennisation des activités des entreprises ainsi que pour leur développement économique, mais elle ne sera source d'avantages concurrentiels que si l'entreprise innovatrice y voit ses comptes en atteignant les objectifs escomptés, à savoir la commercialisation. En effet, pour les réseaux de partenariat entre entreprises et universités, c'est la maitrise de la diffusion des innovations qui constitue la source fondamentale de performance. C'est le rôle privilégié du marketing de l'innovation.

Par ailleurs, les espaces d'innovation collaborative sont considérés par les industriels comme des dispositifs de mobilisation, sur un angle collectif, de projets de R\&D, en profitant des connaissances scientifiques très poussées détenues par les entités de recherche universitaire. Pour les académiques, s'allier avec des entreprises est aussi un moyen de trouver des moyens de financement de leurs 
activités de recherche. Toutefois, de plus en plus d'unités de recherche s'orientent désormais vers une valorisation des résultats de leur recherche vers les marchés (valorisation commerciale), la résolution de problèmes et la satisfaction de besoins socioéconomiques (valorisation sociale). Dans les processus d'innovation collaborative visant à optimiser le développement de nouveaux produits et services, le rôle du marketing est encore plus prépondérant. Parce que l'innovation est comprise et considérée différemment selon la nature et le positionnement des acteurs (entreprise, université, client ou fournisseur). L'entreprise et ses acteurs internes la considèrent comme un moyen à user pour rester en phase avec les attentes du marché, améliorer leurs produits et services, et pérenniser leurs activités. Alors que par leurs activités scientifiques, les chercheurs universitaires espèrent découvrir de nouvelles technologies et faire évoluer les sciences. Et pour les clients, il s'agit de disposer en permanence de produits et de services toujours plus performants et présentant davantage d'originalité. Dès lors, l'innovation favorise également le développement de nouveaux marchés [PRI 03].

\section{La transversalité du Marketing de l'innovation}

De manière générale, le processus d'innovation est jalonné d'étapes interconnectées demandant des allers-retours incessants entre les différents acteurs concernés au sein de l'entreprise [KLI 86; AKR 88]. Il en est de même pour le cas du processus d'innovation collaborative par les interactions université-industrie, un procédé requérant la contribution d'un ensemble d'experts appartenant à des organisations distinctes.

Et dans les deux cas (innovation organisationnelle et innovation inter-organisationnelle), c'est bien le marketing, à travers ses anticipations, qui joue le rôle de guide des acteurs du projet tout au long du processus d'innovation. Ces anticipations se nourrissent notamment des observations du marché, mais peuvent également être issues des stratégies et des représentations internes aux différentes organisations engagées dans le projet collaboratif [DAR 06]. Les compétences liées aux observations du marché sont détenues par les acteurs industriels (entreprises) et interviennent dans le cadre des approches market-pull. C'est-à-dire que dans ce cas de figure, le cadre d'orientation de l'innovation est développé à travers la demande de nouveautés du marché et la recherche de solutions à des problèmes et besoins déjà identifiés. Selon cette approche, c'est la compréhension du marché qui déclenche le processus d'innovation [SCHM 66].

À l'inverse, les stratégies et représentations internes peuvent être considérées comme les capacités techniques et technologiques intrinsèques aux chercheurs et scientifiques (universitaires et ingénieurs). Il s'agit de l'approche technology-push basée sur l'utilisation des rendements de la technologie pour proposer des innovations dérivées des découvertes scientifiques et techniques réalisées [SCHU 34 ; THI 13]. Selon cette considération, c'est la portée des connaissances scientifiques disponibles qui permet de générer de nouveaux produits développés à partir des activités de recherche et développement. De ce fait, le processus ne se base pas ici sur une demande clairement exprimée et identifiée à l'avance, mais sur les possibilités permises par la technologie.

Dans une approche «market pull», le marketing est directement à l'origine du développement de la nouveauté grâce à ses études analytiques ayant pour principal objet de déceler les besoins du marché auxquels elle est amenée à répondre. C'est le marketing amont. Dans une approche «technology push» par contre, le rôle du marketing intervient à la fin du processus d'innovation pour chercher des moyens de commercialisation de la nouveauté, c'est-à-dire l'organisation de la rencontre entre l'innovation générée et une éventuelle demande. C'est le marketing aval.

Ainsi, il apparaît clairement que dans le second modèle dénué d'une orientation marché primitive affichée, l'innovation créée obvie à davantage d'incertitudes que dans le cas des innovations générées selon le premier modèle basé sur la prise en compte préalable des besoins du marché. En effet, l'innovation imposée peut faire l'objet de distance avec le marché, et celle-ci devra donc chercher à trouver son marché et à convaincre des clients potentiels. Ses chances de succès sont donc moindres. 
Pourtant, notre constat est que les innovations issues d'interactions université-industrie ont très souvent fait l'objet de problème d'inadéquation avec la demande dès lors que le projet collaboratif porteur s'était uniquement focalisé sur les aspects technologiques au détriment d'une mise sur les potentialités d'applications marketing. C'est pourquoi nous considérons qu'il est alors fort utile de conférer au marketing un rôle d'intégrateur de l'innovation qui lui permet de situer le client au cœur du processus d'innovation collaboratif, en portant le curseur sur les attentes du marché, et en situant ses contributions au-delà des seules étapes de diffusion et de commercialisation du processus. Il s'agit là de connaître le marché et de faire connaître ses attentes auprès des autres parties prenantes (notamment scientifiques), en jouant un rôle d'intermédiation entre le projet et le marché.

\subsection{Le marketing, intégrateur de l'innovation}

Pour une entreprise considérée isolément (innovation solitaire), le lancement d'un nouveau produit ou service s'accompagne d'un coût financier assez important et d'une prise de risque qui méritent d'être pris en considération. De même, en passant d'un système socioéconomique fondé sur le matériel à un système basé sur la connaissance, les conditions de compétitivité et de performance des entreprises se sont fortement métamorphosées. Couplées aux constats présentés dans les paragraphes précédents, ces observations permettent d'énumérer un certain nombre d'exigences qui imposent à l'entreprise d'aujourd'hui l'adaptation à de nouvelles donnes. Il s'agit notamment de l'ouverture de son processus d'innovation vers l'extérieur afin de se rapprocher de partenaires scientifiques et dans l'optique de contourner des obstacles à l'innovation en ayant accès à des connaissances stratégiques rares, c'est-à-dire celles qui permettent d'innover en levant des verrous technologiques incommodant leurs désirs d'innovation. C'est pour dire qu'à l'époque de la mondialisation des technologies et des marchés, l'innovation solitaire est quasiment impossible.

Cependant, comme évoquées supra, les coopérations entreprises-industries portant sur la combinaison d'aspects technologiques et commerciaux génèrent très souvent des innovations faisant l'objet de problèmes de diffusion et de commercialisation, nécessitant dès lors une meilleure connaissance du marché et des capacités d'anticipation de ses besoins futurs. Dans le processus d'innovation collaborative, il s'agit à la fois d'encastrer des compétences de « savoir » (connaissances) et de «savoir-faire », puis à les combiner avec des capacités de «savoir vendre » intrinsèques aux entreprises. Ces dernières sont indispensables au processus d'innovation lui-même, car permettent à travers le marketing, d'élaborer les conditions de mise sur le marché des innovations issues de la coopération interorganisationnelle.

De manière transversale, le rôle du marketing se rapporte à toutes les phases de l'innovation : idéation, conception, développement, production et diffusion. En tant qu'intégrateur de l'innovation, la fonction marketing se caractérise par une transversalité qui lui octroie une place et un rôle à tous les niveaux du processus. Il est dans la plupart des cas à l'origine des idées innovatrices et évalue leur potentiel commercial. Lors de la gestion du processus d'innovation collaborative, c'est aussi au marketing de faire entendre la «voix du client» en travaillant en étroite collaboration avec les chercheurs pour que les idées d'innovation mises en avant soient en adéquation avec les attentes du marché, et que l'orientation des projets d'innovation collaboratifs prenne en considération le potentiel de commercialisation des technologies en œuvre. Puis, une fois l'opportunité d'innovation prouvée, les activités de R\&D déployées et les prototypes développés; il revient aussi au marketing d'assurer la gestion des tests réalisés auprès des clients, et en dernier rempart, d'élaborer les conditions de mise sur le marché des innovations générées en nouveaux produits ou services.

Dans une organisation considérée isolément, les différentes phases d'un projet d'innovation sont occupées par des acteurs internes qui chacun, joue un rôle de «représentant» du client dans son domaine de compétence. Par exemple, l'ingénieur pour les aspects techniques, le designer pour l'usage et l'agrément, le financier pour les prix, etc. [FER 06]. Pour la fonction marketing de l'entreprise, il 
s'agit de garantir un suivi de tout le processus d'innovation par l'exercice d'une fonction transversale aux étapes de la conception, du développement et de la fabrication du produit, ou de la réalisation du service. Elle travaille de concert avec les autres fonctions de l'entreprise amenées à contribuer au déploiement du processus d'innovation.

Par contre, dans un réseau de partenaires animés par des velléités d'innovation collaborative, les rôles sont détenus par différents acteurs appartenant à des organismes disparates et occupant des périmètres d'intervention différents, ce qui peut rendre complexe le processus d'innovation. Pour atténuer cette complexité et remédier aux problèmes de succès des innovations issues d'un groupement de partenaires qui, au demeurant, aspirent pourtant à une coopération bénéfique à des externalités et des complémentarités positives, les projets d'innovation collaborative ont besoin, en compléments des savoir-faire techniques et technologiques, de compétences de management porté par une équipe de pilotage. Cela permet aussi, entre autres, de réduire les tensions auxquelles sont confrontées les parties prenantes. L'avantage d'une structuration séparée permet d'optimiser la collaboration entre des parties prenantes hétérogènes et d'éviter les écueils relatifs aux intentions stratégiques antinomiques.

Dans ce cadre, le rôle de la cellule d'animation et de pilotage est de manager le projet collaboratif et d'assurer la gestion de la coordination des efforts destinés à permettre la génération d'innovations propices à l'accès aux marchés. Dans le contexte d'innovation collaborative par les interactions université-industrie, les possibilités d'accès aux marchés sont permises par l'apport des acteurs industriels à travers l'orientation client qu'ils promeuvent et la connaissance qu'ils ont du marché et de ses attentes. Pour ces derniers, collaborer avec d'autres partenaires pour innover est un moyen de surmonter des problèmes d'inefficacités et de gagner en performances en matière d'innovation, en bénéficiant notamment des connaissances scientifiques des acteurs universitaires.

Le rôle de la structure d'animation et de pilotage est de permettre cette infusion de capacités managériales et de compétences marketing. Elle a en outre à veiller à l'instauration d'une vision partagée autour de l'innovation, car la coopération interorganisationnelle implique un degré d'implication des parties prenantes dans les processus d'innovation et éventuellement le partage d'informations et de ressources stratégiques.

\subsection{Le marché en tant que vecteur de la transversalité du marketing}

Le marketing de l'innovation est une démarche d'interactions, tout comme le concept d'innovation collaborative. Pour le premier, il s'agit d'une part d'assurer l'interaction entre le marché et le projet collaboratif, et d'autre part d'assurer l'interaction entre lui-même et les autres acteurs engagés dans le projet. Ainsi, il constitue un chainon à l'intersection de ces trois univers afin de faciliter, pour l'ensemble des parties du projet d'innovation collaboratif, la compréhension d'aspects multidimensionnels touchant les dimensions techniques et technologiques, mais aussi les dimensions économiques et psychosociologiques. Pour ce faire, il base son principe fonctionnel sur la cohérence du projet et de l'innovation visée, en considérant le marché comme le fil conducteur de ses actions de centralisation du processus d'innovation collaborative.

Dans la vie des entreprises, le marché (d'une manière générale et le besoin du client d'une manière plus spécifique), représente le point de filtres des activités du processus d'innovation. Il occupe tous les acteurs de l'entreprise impliqués et qui œuvrent pour sa rentabilité par la proposition de produits ou services qui répondent aux attentes du marché et satisfassent les besoins des clients.

Dans les espaces d'innovation collaborative promouvant les interactions entre universités et industries, l'innovation est le fruit de la coopération et représente, au bout du compte, le résultat escompté par les acteurs. De ce fait, il apparaît indispensable d'avoir dans la pratique, des acteurs impliqués, ayant un engagement concret et interagissant autour d'objectifs partagés. Certes, les intérêts inter-organisationnels ne sont pas mutuellement exclusifs et les motivations des acteurs diffèrent, mais 
c'est plus une contribution au renforcement de la valeur de la collaboration universités-industries, à condition qu'il y ait une posture culturelle et une réglementation qui incitent à l'instauration d'un environnement plus ouvert et collaboratif. Pour cela, il faut nécessairement l'adoption d'une approche plus intégrale de la part des deux parties, consistant à les rapprocher par l'orientation marché des projets.

Dans ce contexte, le marketing de l'innovation a aussi un rôle de fédération de l'ensemble des représentations complémentaires juxtaposées au sein du projet collaboratif. Il permet l'intercompréhension entre les acteurs et leur adhésion à un objectif commun qui mène vers le marché. Il est chargé, en cas de nécessité, d'infléchir les trajectoires de l'innovation vers des modalités d'applicabilité sur le marché, donc à destination d'une demande potentielle.

Toutefois, la focalisation sur les aspects liés au marché n'occulte pas l'exploitation et l'optimisation des possibilités de la technologie et la prise de risque inhérente à toute l'innovation. En effet, cela pourrait conduire à l'instauration d'une logique de dépendance vis-à-vis des seules représentations des clients au détriment d'une génération d'innovations de ruptures pourtant indispensables et déterminants pour la survie des entreprises et pour l'avancée de la science et des technologies.

De manière concrète, les universités doivent s'engager à travailler avec les industriels en imprégnant davantage leur propre culture de l'esprit des entreprises, et les industriels se doter de services de gestion des relations avec les universités. Dans ce cadre, l'élaboration d'indicateurs d'implication [ALI 10] renvoyant à l'implication et à la participation des membres de l'espace collaboratif permettrait d'optimiser les résultats d'innovation. Cette implication se mesure par l'intention de collaboration et les actions concrètement effectuées par les membres se traduisant par une participation à des projets, aux actions d'animation et aux instances de la gouvernance [LAL 13].

De manière synthétique, l'innovation collaborative apparaît comme le résultat d'un système complexe d'interactions entre des industriels et des universités cohabitant dans des projets collaboratifs, tout en étant animés par des objectifs disparates, donc une dynamique complexe d'acteurs/projets. Dans ce contexte, l'innovation collaborative performante est basée sur une approche hybride qui se focalise sur la dimension technologique tout en intégrant les aspects "marché » [SLA 99, SLA 00 ; JAW 96, JAW 93 ; WEB 94 ; LAM 05, THI 13]. L'introduction d'innovations dans les marchés s'accompagne toujours d'incertitudes et un risque de non-adoption par les consommateurs. En conséquence, la commercialisation d'innovations et d'idées intéressantes mérite d'être formellement encadrée par le markéting afin d'assurer leurs succès et qu'elles soient sources de création de valeurs pour les parties prenantes.

Du point de vue de l'objectivation socio-sémantique, les espaces d'interaction industries-universités sont des lieux de communautés épistémiques qui se doivent d'une part d'impliquer une orientation «marché », et d'autre part une orientation «technologie » (standards technologiques) partagées par les partenaires pour influencer favorablement le succès de l'innovation collaborative.

\section{Conclusion}

Alors que se développent de manière persistante les espaces d'innovation organisés autour d'interactions entre industries et organismes de recherche universitaire demeurent des difficultés d'accès aux marchés des innovations générées à leur sein. C'est spécifiquement le cas des pôles de compétitivité français [THI 13]. Nonobstant, la littérature a peu étudié les activités en aval du processus d'innovation (diffusion et commercialisation). Elle s'est surtout intéressée aux activités en amont, à savoir comment optimiser la collaboration interorganisationnelle visant à obtenir et intégrer de nouvelles sources d'innovation. C'est pourquoi, le succès d'une innovation étant plus lié à son adoption par le marché, un regain d'intérêt pour la commercialisation et l'adoption des innovations s'intensifie actuellement. 
Aujourd'hui, le marketing de l'innovation est incontournable pour optimiser la diffusion des innovations issues de collaborations entre la recherche et les entreprises. Les réseaux d'innovation qui en maîtrisent les rouages peuvent surveiller à temps réel les évolutions du cadre macroéconomique autour des technologies et du marché : besoins et attentes des consommateurs, concurrence, connaissances scientifiques... Il joue un rôle transversal entre les différents profils d'acteur de l'innovation collaborative (managers, technicien, chercheurs et scientifiques) et se sert de la variable «orientation marché » comme le fil conducteur de ses actions visant l'instauration d'une cohérence d'ensemble et la facilitation de l'accès aux marchés des innovations générées (diffusion et commercialisation).

Le schéma organisationnel classique des processus d'innovation collaborative s'apparente à celui des innovations issues des laboratoires de recherche, avec un détachement vis-à-vis des marchés et un pilotage sous l'esquisse d'un chef de projet dénué de connaissance des aspects marketing. C'est l'approche «technology push», alors que le processus d'innovation est fait d'une combinaison de savoirs et de compétences requérant, pour être efficiente, l'exercice d'une gestion transversale de l'ensemble de ses phases d'élaboration. Il semble alors plus pertinent d'octroyer au marketing le rôle de «chef d'orchestre » [DAR 06] afin de garantir un aboutissement du management de l'innovation jusqu'à sa diffusion sur le marché.

Dans ce contexte, les espaces d'interactions universités-industries doivent considérer «l'orientation marché » comme un vecteur stratégique permettant de rapprocher les deux acteurs autour d'objectifs communs de diffusion et de commercialisation des innovations, en minimisant les écueils générés par les tensions et les différences de perception et d'intérêt vis-à-vis des aspects "marché » et «technologies». Ils doivent faire l'effort de se comprendre et d'avoir une connaissance à la fois des aspects technologiques et des questions liées aux problématiques des marchés.

Le développement d'innovations dans les espaces d'interaction universités-industries a fait émerger de nouveaux concepts situés au carrefour entre innovation et marketing : open innovation, innovation fermée, innovation réseau, rôle des acteurs internes et externes dans le succès de l'innovation collaborative...

\section{Bibliographie}

[ACR 88] AKRICH M., CALlON M., LATOUR B., «A quoi tient le succès des innovations : l'art de l'intéressement », Gérer et Comprendre, Annales des Mines, (11), p.4-17., 1988.

[ALI 10] Aliouat B., (dir.), Les Pôles de compétitivité : Performance et Gouvernance des réseaux d'innovation, Lavoisier-Hermes Sciences Publishing, Paris, 2010.

[ALV 10] Alves S, Gosse B, SPRIMONT P-A., «Dimensions et conséquences de la satisfaction au travail des apprentis : le cas de l'enseignement supérieur », Revue Internationale de Psychosociologie, novembre, 2010.

[AYD 86] AYDALOT P., (ed), Milieux innovateurs en Europe, Paris, GREMI, 1986.

[BEA 12] BEARING POINT-ERDYN-TECHNOPOLIS ITD, Étude portant sur l'évaluation des pôles de compétitivité, Rapport global, 15 juin 2012.

[BEC 79] BECATTINI G., «Dal settore industriale al distretto industriale. Alcune considération sull'unità di indagine dell'economia industriale », Rivista di Economia e Politica Industriale, 2, 7-21, 1979.

[BEL 05] BELL G.G., « Clusters, networks, and firm innovativeness Strategic », Management Journal, 26, p. 287, 2005

[BOC 06] BoCQUET J.-F., PIZZI A., RESCH L., «Full-scale, Industrial wood floor assembly and structures by weldedthrough dowels », J.Adhesion Sci. Technol, in press, 2006.

[BOC 09] BoCQUET R., MOTHE C., « Gouvernance et Performance des pôles de PME », Revue Française de Gestion, vol. 35/190, pp. 101-122, 2009.

[BRE 15] BREM A., VIARDOT É., Adoption of Innovation: Balancing Internal and External Stakeholders in the Marketing of Innovation, In: BREM A., VIARDOT É. (eds) Adoption of Innovation. Springer, Cham, 2015. 
[CHA 10] Chabault D., «Gouvernance et trajectoire des pôles de compétitivité », Management \& Avenir, vol. 6, n 36, p. 130-151, 2010.

[CHAB 09] CHABAULT D., Gouvernance et trajectoire des réseaux territoriaux d'organisations : une application aux pôles de compétitivité, Thèse de Doctorat en Sciences de Gestion. Tours : Université François Rabelais, 2009.

[CHAL 09] Chalaye S., MASSARD N., «Les clusters, diversité des pratiques et mesures de performance», Revue d'économie industrielle - $\mathrm{n}^{\mathrm{o}} 128$, 4ème trimestre 2009.

[CHE 03] CheSBROUGH H.W., Open innovation: The new imperative for creating and profiting from technology, Harvard Business school Pess, Boston, 2003.

[CMI 08] CM InTERNATIONAL ET BCG, L'évaluation des pôles de compétitivité 2005-2008, La Documentation française, Paris, 2008.

[COO 98] COOKE P., URANGA M.G., ETXEBARRIA G., « Regional systems of innovation: an evolutionary perspective », Environment and Planning A, 30, pp. 1563-1584, 1998.

[DAR 06] DARENE N. ET ROMON F., «La représentation du client futur dans la construction d'une offre innovante, Une étude du rôle du Marketing », Annales des Mines, Série Gérer et Comprendre, Numéro 83, Mars, pp. 36-46, 2006.

[DAV 91] Davis D., Morris M., ALlen J., «Perceived environmental turbulence and its effect on selected entrepreneurship, marketing, and organizational characteristics in industrial firms », Journal of the Academy of Marketing Science, 19 (1), pp. 43-51, 1991.

[DAY 90] DAY G.S., Market-Driven Strategy, Free Press, New York, 1990.

[DAY 94] DAY G.S., «The Capabilities of Market-Driven Organizations », Journal of Marketing, Vol. 58, N $\mathrm{N}^{\mathrm{O}}$ 4, pp. 37 $52,1994$.

[DEF 10] De Faria P., Lima F., Santos R., « Cooperation in Innovation Activities: The Importance of Partners », Research Policy, 39 (8), pp. 1082-1092, 2010.

[DES 93] DeShPANDÉ R., FARLEY J.U., WEBSTER JR F., « Corporate culture, customer orientation, and innovativeness in Japanese firms: a quadrad analysis », Journal of Marketing 57(1): 23-37, 1993.

[DES 97] DeSTOT M., ETI et PME : Pour une innovation compétitive, Les Essais, Fondation Jean Jaurès, 2011.

[EDQ 97] EDQUIST C., Systems of Innovation: Technologies, Institutions, and Organizations, Londres, Pinter, p. 1 (traduction), 1997.

[FER 2006] Fernez-Waleh, S. ET Romon, F., Management de l'innovation : De la stratégie aux projets, Edition Vuibert, 2006.

[GOG 15] GOGLIO-PRIMARD K., CRESPIN-MAZET F., « Organizing Open Innovation in Networks - the role of boundary relations », Management International, Volume 19 - Numéro special — p. 135-147, 2015.

[GRA 85] GRANOVETTER M., « Economic-Action and Social-Structure - The problem of Embeddedness », American Journal of Sociology, Vol. 91, p. 481-510, 1985.

[JAW 96] JAWORSKI B.J., KoHLI A.K., « Market orientation: Review, Refinement, and Roadmap », Journal of Market Focused Management, 1, pp. 119-135, 1996.

[JAW 93] JAWORSKI B., KOHLI A.K., « Market orientation: antecedents and consequences, Journal of Marketing », 57, 3, pp. 53-70, 1993.

[JOH 11] JOHnSOn G., WhitTington R., SchOles K., FréRy F., Stratégiques, Pearson Education, 9e edition, 2011.

[KLI 86] Kline S., Rosengberg N., An overview of innovation, Landau R., Rosenberg N. (eds), The Positive Sum strategy, National Academy Press, Washington, 1986.

[KOH 93] KOHLI A.K., JAWORSKI B.J., KUMAR A., « MARKOR: A measure of market orientation », Journal of Marketing Research, 30 (4), 467-477, 1993.

[KOH 90] KOHLI A.K., JAWORSKI B.J., « Market orientation: The construct, research propositions, and managerial implications », Journal of Marketing, 54 (2), 1-18, 1990.

[LAL 13] LALLEMAND, A.S., L'évaluation de la performance des réseaux territorialisés d'organisations : le cas des pôles de compétitivité français, Thèse en sciences de gestion, Université Paris 2 Assas, 474p., 2013.

[LAM 05] Lambin J.-J., Chumpitaz R, De Moerloose C., Marketing Stratégique et Opérationnel, Du Marketing à l'Orientation Marché, 6ème édition, Dunod, Paris, 2005. 
[LEN 15] Le Nagard E., Manceau D., Morin-Delerm S., Le Marketing de l'Innovation, Post-Print, halshs-01186378, HAL., 2015.

[LOU 11] LOUBARESSE E., «Profil généraliste ou profil technique, quand les compétences des pilotes opérationnels influencent le pilotage des Systèmes Productifs Locaux », Management \& Avenir 10/2011 ( $\mathrm{N}^{\circ}$ 50), pp. 207-225, 2011.

[LOU 08] LOUBARESSE E., «Influence des caractéristiques des pilotes de réseaux locaux d'organisations sur leurs rôles », Revue Management International, pp. 85-96, 2008.

[MAI 031] MAILlat D., « Développement des systèmes territoriaux de production, compétitivité et innovation, Acte du colloque international de développement local: le développement local dans la perspective du développement humain », Campo Grande, Brésil 25-28 novembre 2003.

[MAR 90] Marshall A., Principes d'économie politique, Paris, Giard et Brière, 1890 (1ere édition française, 1906).

[NAR 901] NARVER J.C., Slater S.F., "The Effects of a Market Orientation on Business Profitability », Journal of Marketing, Vol. 54 (October), pp. 20-35, 1990.

[OEC 031] OECD, The Measurement of Scientific and Technological Activities: Guidelines for Collecting and Interpreting Innovation Data: Oslo Manual, Third Edition by Working Party of National Experts on Scientific and Technology Indicators, OECD, Paris, 2005.

[POR 04] PORTER M.E., Building the Microeconomic Foundations of Prosperity: Findings from the Business Competitiveness Index', in M.E. PORTER, K. SCHWAB, X. SAlA-I-MARTIN AND A. LOPEZ-Claros (eds.), The Global Competitiveness Report 2004-2005, Palgrave Macmillan, World Economic Forum: New York, pp: 19-50, 2004.

[POR 00] PORTER M.E., Locations, Clusters, and Company Strategy, In: Clark G.L., Feldman M.P. et Gertler M.S. (eds.), The Oxford Handbook of Economic Geography, Oxford: Oxford University Press, 253-274, 2000.

[POR 98] PORTER M.E., Clusters and the new economics of competition, Harvard Business Review, pp. 76, 77-90, 1998.

[POR 90] PORTER M.E., The Competitive Advantage of Nations, London, Mac Millan, 1990.

[PRI 03] PRIME, N. ET UsUNIER, J. C., Marketing international-Développement des marchés et management des hommes, Paris, Edition Vuibert, 2003.

[POW 90] POWELL W.W., « Neither market nor hierarchy: Network forms of organization », Research in organizational behavior, 12, pp. 295-336, 1990.

[PUE 92] RUEKERT, R. W., «Developing a market orientation: An organizational strategy perspective », International Journal of Research in Marketing, 9(3), 225-245, 1992.

[RET 09] RETOuR, D. dir., «Dossier "Pôles de compétitivité” », Revue Française de Gestion, vol. 35, n 190, janvier, pp. 91-209, 2009.

[SAL 13] SALMELIN, B., «Innovation in Horizon 2020-Reflections from Open Innovation 2.0 paradigm », Open Innovation 2.0 Conference, Brussels, November 28, 2013.

[SAX 94] SaXenian A.L., Regional Advantage: Cultureand Competition in Silicon Valley and Route 128, Cambridge, MA: Harvard University Press, 1994.

[SCHM 66] SCHMOOKLER J., Invention and Economic Growth, Cambridge, MA: Havard University Press, 1966.

[SCHU 34] SCHUMPETER J.A., The Theory of Economic Development, Cambridge, MA, US: Harvard University Press, 1934.

[SLA 99] SLATER S.F., NARVER J.C., «Market-oriented is more than being customer led, Strategic », Management Journal, 20 (1999), pp. 1165-1168, 1999.

[SLA 00] SLATER S.F., NARVER J.C., «The positive effect of a market orientation on business profitability: A balanced replication », Journal of Business Research, 48, pp. 69-73, 2000.

[STO 88] STORPER M., SCOTT A., The Geographical Foundations and Social Regulation of Flexible Production Complexes, in: Wolch J., Dear M., eds, The Power of Geography, London, Allen and Unwin, 1988.

[THI 18] THIAW C. A. L., l'innovation localisée pour le développement économique du Sénégal, Édition Harmattan, Paris, 2018.

[THIa 17] THIAW C. A. L., Innovation collaborative au Sénégal : pour une coévolution entre science, technologies et innovation, Congrès AEI Dakar, 6, 7 et 8 Décembre 2017, Dakar, Sénégal, 2017. 
[THIb 17] THIAW C. A. L., AliouAT B., Analyse comparative des écosystèmes d'affaires pour impulser l'innovation et la compétitivité en Afrique : proposition d'un modèle idoine pour le Sénégal, SERGe Days, 5ème édition, 27-29 Avril 2017, Saint-Louis, Sénégal, 2017.

[THI 16] Thiaw C., Aliouat B., Entrepreneuriat en équipe et vision partagée : Le cas des pôles de compétitivité, in Paturel Robert, Levy Thierry : L'entrepreneuriat en équipe, Presses Universitaires de Provence, 2016.

[THI 13] THIAw C. A. L., Innovation collaborative et orientation client/marché au sein des Pôles de compétitivité : Analyse empirique des dynamiques de projets/acteurs (Cas des Réseaux d'innovation en Région PACA), Thèse de doctorat en Sciences de gestion, Université Nice-Sophia Antipolis, Avril, 2013.

[THI 11] THIAW C., Marketing de l'innovation et benchmarking au sein des pôles de compétitivité, in Aliouat B., (dir.), Les pôles de compétitivité, gouvernance et performance des réseaux d'innovation, Traités IC2, Traité Technologies et Développement durable, Publications Hermes Science, Lavoisier, Paris, 2010, 448 p., 2011.

[THI 09] Thiaw C., Aliouat B., «L'encastrement de compétences d'innovation au sein des pôles de compétitivité : savoir, savoir-faire et savoir vendre », Colloque international, Pôles de compétitivité et développement régional, HEC Management School, Liège, mars, 2009.

[TOR 15] TORRE \& ZIMMERMAN, «Des clusters aux écosystèmes industriels locaux», Revue d'économie industrielle 2015/4 (nº 152), pages 13 à 38, 2015.

[VIC 16] VICENTE J., Économie des clusters, Repères Économie nº 676 - 128 pages, 2016.

[VON 16] Von Pechmann F., Chamaret C., Parguel B., Midler C., «Comment prévoir le succès d'une innovation de rupture : le cas du véhicule électrique », Décisions Marketing, 81, 81-98, 2016.

[WEB 66] WEBSTER A., «International evaluation of academic - industry relations: contexts and analysis », Science and Public Policy, 21 (2), pages 72-78, 1994. 